\title{
Curvature effect in grazing $X$-ray reflectometry
}

\author{
F. Bridou \\ Institut d'Optique Théorique et Appliquée, Laboratoire Associée du CNRS, B.P. 147, \\ 91403 Orsay Cedex, France
}

(Recen'ed 19 November 1993, revised 7 April 1994, accepted 4 May 1994)

\begin{abstract}
Résumé. - La réflectométrie en $X$ rasants est utilisée couramment pour la caractérisation d'empilements de couches minces. Les paramètres à détermıner sont : épaisseurs, rugosités et indices. On peut y accéder en ajustant la courbe expérimentale de réflectivité avec une courbe théorıque calculée à partir de ces paramètres. Dans cette approche, on suppose que l'échantillon est plat. On peut montrer expérimentalement que la courbure de l'échantillon modifie la réflectivité. Un exemple d'échantillon à deux courbures (concave et convexe) dans deux directions perpendiculaires montre le type d'écart de réflectivité, puis d'analyse que l'on peut obtenir, lorsque l'échantillon est placé dans un sens, puis dans la dırection perpendiculaire. Afın d'étudier quantitativement l'effet de la courbure, cinq paires d'échantillons calibrés de silice revêtus de couches identiques de TiN ont été réalisées. L'étude théorique montre que pour un montage donné, la courbure de l'échantillon modifie l'ouverture du faisceau réfléchi, notamment dans le plan d'incidence, alors qu'elle est négligeable dans le plan perpendiculaire au plan d'incidence. Cette varıation d'ouverture en fonction de l'incidence entraîne des variations significatıves de l'intensité mesurée, tenant compte des caractéristiques géométriques de l'appareil de mesure. Un modèle de calcul a été réalisé, et un bon accord a été observé avec les courbes expérimentales, notamment dans la région du plateau de réflectivité.
\end{abstract}

\begin{abstract}
Grazing X-ray reflectometry is currently used for the characterization of thin layer stacks. The parameters to be determined are thickness, roughness, and optical index. They can be worked out by fittıng the recorded reflectivity curve, with a theoretical one calculated with the appropriate parameters. In such a calculation, the sample is supposed to be flat. It can be shown experimentally that the curvature of the sample modifies the expected reflectivity. An example of a saddle shaped sample, with opposite main curvature in two perpendicular directions shows typical differences on recorded curves for these two perpendicular directions. In order to make a quantitative study of the effect of the sample curvature, five pars of spherical silica substrates have been made and coated with similar TiN layers. A theoretical study has also been made. It is shown that, for a given set-up, the sample curvature changes the aperture of the reflected beam with respect to that of the incident beam. At grazing incidence, the aperture change ts significant in the incidence plane, while it can be neglected in the plane perpendicular to the incidence plane. As a consequence of the aperture change, the recorded intensity can be increased or decreased. depending on the position of the source image with respect to the position and width of the stop aperture in the image space. A calculation has been made, taking into account the geometrical characteristics of the equipment. The results have been compared with the reflectivity curves measured for the TiN layers deposited on the curved silica substrates. It can be seen that the anomalies in the reflectivity curves, induced by the substrate curvature in the plateau region are well accounted for by the model.
\end{abstract}




\section{Introduction.}

Grazing X-ray reflectometry is used in order to study stacks of thin layers films [1, 2].

A sketch of the IOTA (Institut d'Optique Théorique et Appliquée) set-up is given in figure 1. The source unit is an $\mathrm{X}$-ray source with a copper anode. The $\mathrm{K} \alpha_{1}$ radiation $(\lambda=0.15405 \mathrm{~nm})$ is obtained by means of a $200 \mathrm{LiF}$ crystal. The sample is positioned on a fixed stand, the source unit and the detector are fixed on arms which move around the same axis. The reflectivity curve is obtained by varying the grazing angle while tracking the reflected beam. An experimental reflectivity curve is obtained and can be fitted with a calculated one. Much information on each layer can be reached by this method, in order to get an index profile of the stack. But, this supposes that the best experimental conditions are satisfied, in particular that the sample is flat. When it is not the case, the analysis of the reflectivity curve can lead to erroneous results.

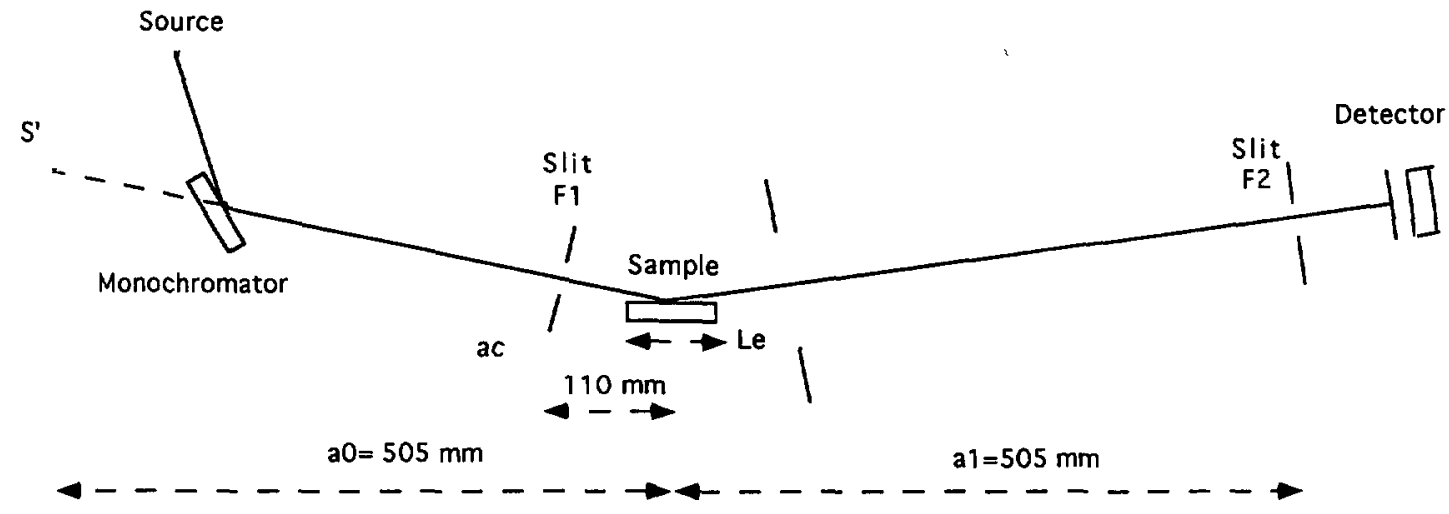

Fig. 1. - IOTA X-ray goniometer sketch.

EXAMPLE OF THE EFFECT OF A CURVED SUBSTRATE ON THE REFLECTIVITY CURVE OF A THIN FILM. - We have examined a saddle shaped sample, the interferogramm of which is given in figure 2. It can be seen that the sample has a concave shape in one direction and a convex one in the perpendicular direction. The concave radius of curvature equals $564 \mathrm{~m}$ and the convex

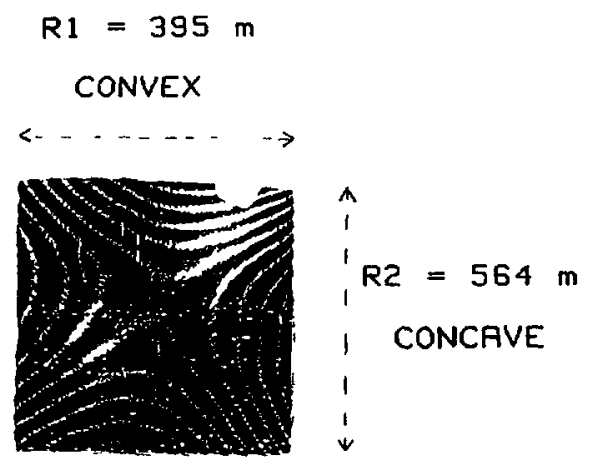

Fig. 2. - Fizeau interferogram of a saddle shaped sample. 
one $395 \mathrm{~m}$. Figure 3 a plots the reflectivity curve recorded on IOTA equipement in those two perpendicular directions. It appears that there are significant differences, in particular in the plateau region, but that the fringe positions are nearly the same.

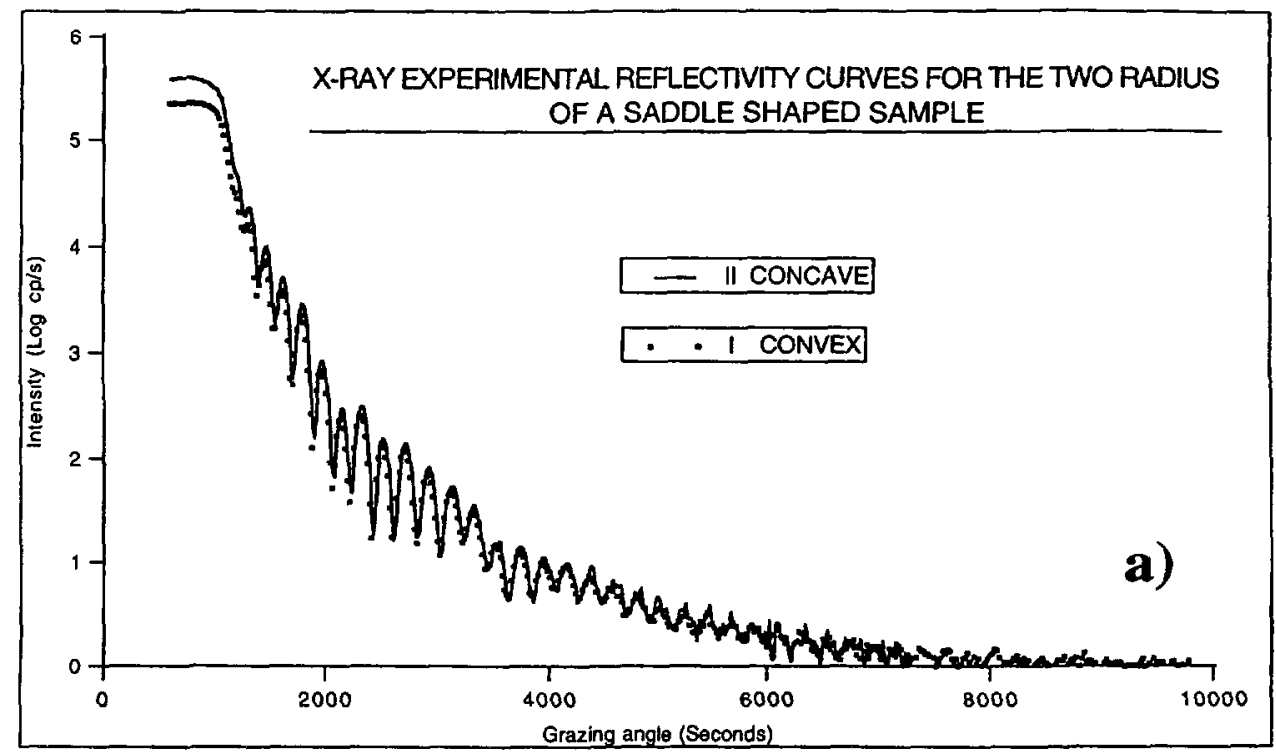

THE TWO PROFILES CORRESPONDING TO THE FIT OF THE TWO REFLECTIVITY CURVES OF THE SADDLE SHAPPED SAMPLE

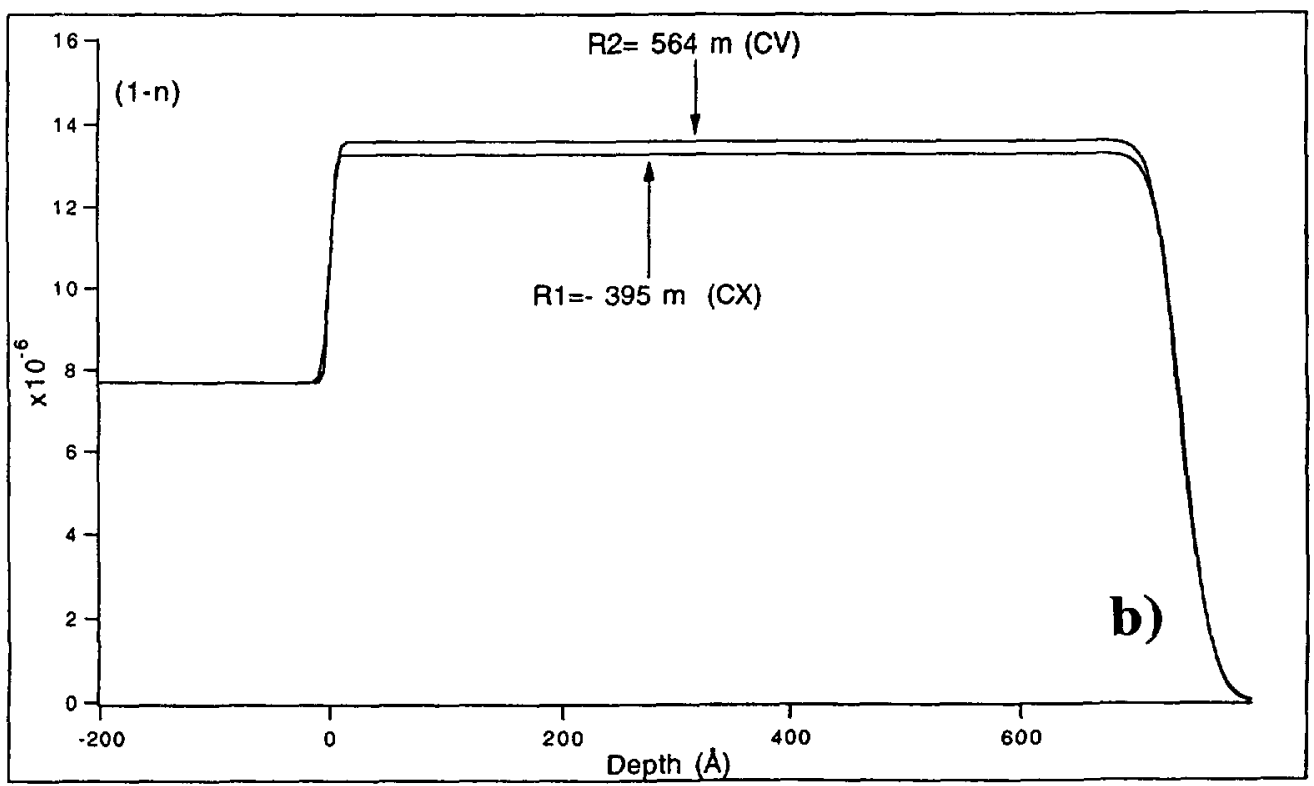

Fig. 3. - a) Experimental reflectivity curves obtained with the saddle shaped sample positioned in the goniometer in two perpendicular directions. b) Corresponding index profiles obtained after the fit of the two curves. 
The two curves are fitted, and the resulting parameters are different, as can be seen on the corresponding index profile (Fig. 3b) calculated with the model described in reference [3].

This example shows that, even if the total layer thickness can be given with good accuracy, when a stack of more than one layer has to be studied, the fact that the sample is not flat can lead to bad index profile. In particular, it is possible to find ghost superficial layers, or false high roughness.

There is another effect due to the curvature of the sample: because the grazing angle $\theta$ on the sample is not constant from one side to the other, the measured intensity is related to a bandwidth $\Delta \theta$ of grazing incidence. It results in a decreasing angular resolution in the experimental reflectivity curve. This effect is not detailed here.

\section{Study of calibrated sample.}

In order to make a quantitative study of the curvature effect, a set of five pairs of calibrated curved samples have been made. The samples are silica glass coated with similar titanium nitride layers, and the radii of positive and negative curvatures measured by optical interferometry $(\lambda=0.6328 \mu \mathrm{m})$ are $1200 \mathrm{~m}, 400 \mathrm{~m}, 100 \mathrm{~m}, 50 \mathrm{~m}$. and $20 \mathrm{~m}$, with an uncertainty of the radius of curvature $|\Delta R / R|<110^{-2}$.

We measured the reflectivity curves of these samples.

2.1 AdJUSTMENT OF THE APPARATUS. - The first procedure consists of tracking the direct beam, while the source is fixed. The recorded intensity curve has a Gaussian shape (Fig. 4). The variation $\Delta \theta$ defined by the aperture of the incident beam, is $0.2 \mathrm{mrad}$ (or $4 l^{\prime \prime}$ ) for the actual width of $120 \mu \mathrm{m}$ of the entrance slit F1 (the exit slit F2 have the same width). For that small range of aperture, it can be seen in figure 4 that the intensity is close to the maximum.

During the setting phase, the source is fixed, the grazing incident beam on the sample is somewhere in the total reflection area. The next step of the adjustment procedure consists in tracking the reflected beam. The intensity curve of the reflected beam has also a Gaussian shape, but in figure 5 , in order to define the maximum with accuracy, the y coordinate is expanded.

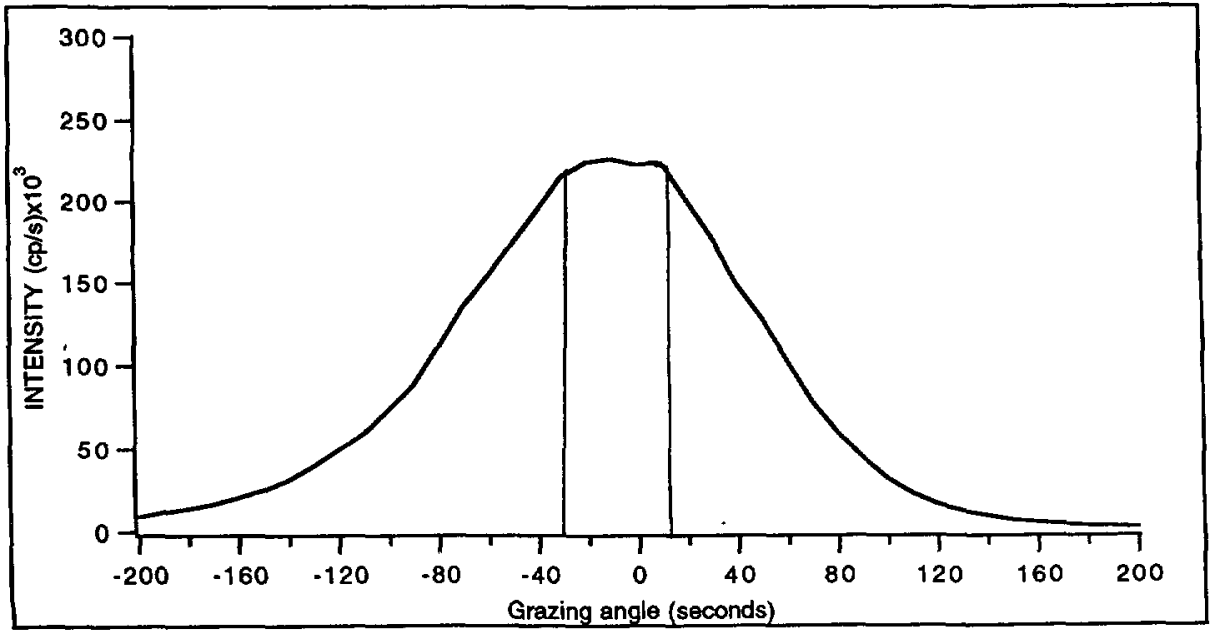

Fig. 4. - Intensity curve of the direct beam recorded on the IOTA set-up in the geometrical conditions of the later measurements. 


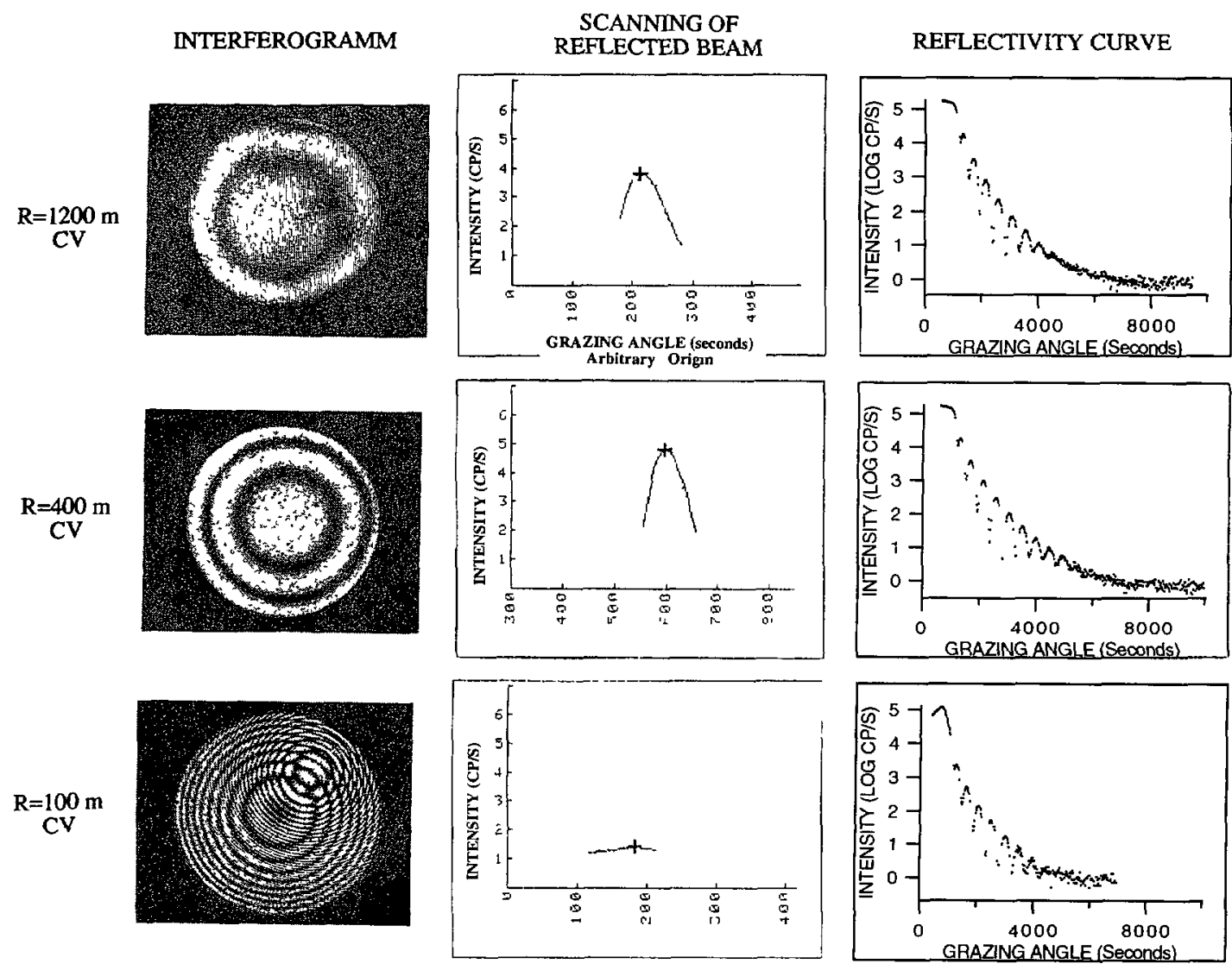

Fig. 5. - Effect of the curvature on the reflected beam geometry. The $y$ coordinate of intensity is in arbitrary units, it is expanded in order to define the maximum with accuracy.

When the curvature of the sample increases, it can be seen that the shape of the intensity of the reflected beam varies, and can become very flat. In this case, the procedure of adjustment is ambiguous and can lead to a bad setting. This is one of the possible effects caused by a curved sample.

2.2 RECORDING OF THE REFLECTIVITY CURVE. - The experimental reflectivity curves given by IOTA set-up show that the fringe positions are nearly the same, but that the intensities are different, and that the plateaux do not have the same shape. For the concave samples (Fig. 6), these effects of curvature seem to be much more important. The curve related to the sample with a radius of curvature of $20 \mathrm{~m}$ has a very bad distorded shape, which is probably due to a bad adjustment as explained above.

\section{Geometrical aberration introduced by a curved sample.}

When a sample is flat, it works like a perfect mirror, and after reflection, the light seems to come from the source image which is obtained by symmetry with respect to the plane of the mirror. 


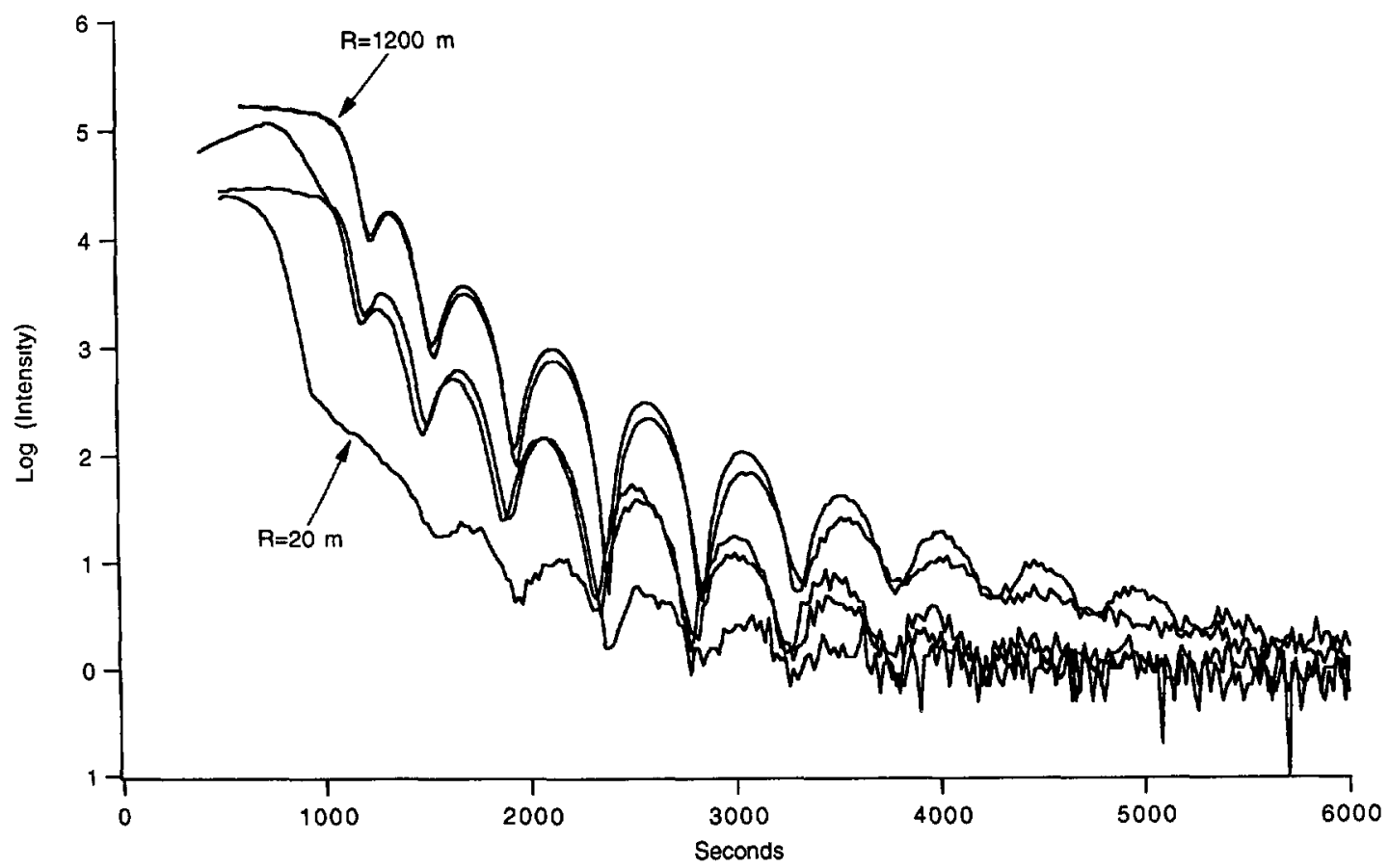

Fig. 6. - Experimental reflectıvity curves recorded on the IOTA set-up with the concave samples.

For a curved sample, an astigmatic aberration occurs [4], and the source image has not the same position (Fig. 7). For the beam section perpendicular to the incidence plane, the position of the source image $S_{1}$ is defined by the relation between the positions of the sagittal focus $S_{0}, S_{1}$.

$$
1 / S_{0}+1 / S_{1}=2 * \sin \theta / R
$$

where $S_{0}$ is the distance between the source and the sample, $S_{1}$ the distance between the image of the source and the sample, $R$ the radius of curvature, and $\theta$ the grazing incidence angle. $S_{1}$ is stationary because the ratio $\sin \theta / R$ is close to zero.

For the beam section parallel to the incidence plane, this relation becomes for the tangential focus $T_{0}, T_{1}$ :

$$
1 / T_{0}+1 / T_{1}=2 /(\sin \theta * R) .
$$

It is shown that the source image position varies with the radius of curvature and also with the grazing incidence $\theta$. For IOTA set-up, figure 8 represents the variation of the position $T_{1}$ of the source image with the grazing incidence angle, for some concave and convex radii. For a flat sample, this position is constant and leads to a straight line. In other cases, in particular for concave radii, $T_{1}$ tends toward infinity and large discontinuities appear. The severe modifications occurring near the plateau region can be explained by these large variations of the source image position with the grazing incidence angle.

\section{Calculation of the recorded intensity variations related to the change of the aperture.}

During the reflectivity recording, the detector is always positioned in the direction of the specularly reflected beam. For the entrance aperture $\Delta \theta=41$ ", in a first approximation, the 


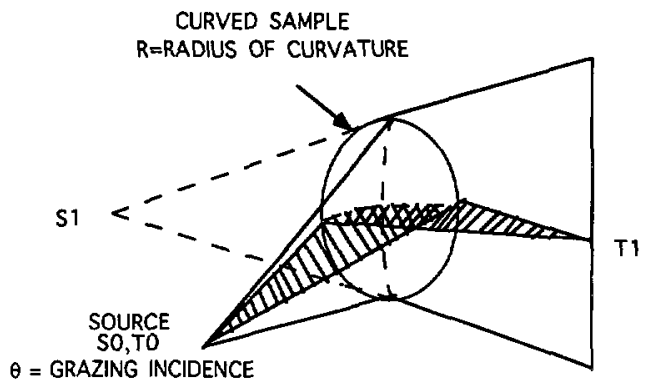

FOR THE BEAM PERPENDICULAR TO THE INCIDENCE PLANE THE DISTANCE S1 BETWEEN THE IMAGE OF THE SOURCE AND THE SAMPLE IS DEFINED BY THE EQUATION :

$$
\frac{1}{S 0}+\frac{1}{S 1}=\frac{2 \operatorname{Sin} \theta}{R} \quad \begin{aligned}
& \text { So, S1 \# SO } \\
& \text { SI SSSTATIONARY }
\end{aligned}
$$

FOR THE BEAM PARALLEL TO THE INCIDENCE PLANE THE DISTANCE T1 BETWEEN THE IMAGE OF THE SOURCE AND THE SAMPLE IS DEFINED BY THE EQUATION :

$$
\frac{1}{T_{0}}+\frac{1}{T_{1}}=\frac{2}{R \operatorname{Sin} \theta} \quad \text { IIVARIES WITH R AND } \theta
$$

Fig. 7. - Astigmatıc aberration resulting from a curved sample under grazing incidence.

variation of the intensity with $\theta$ is neglected in our calculation. Then the measured flux can be defined by :

$$
\mathrm{d} \phi=I(\theta) . \mathrm{d} \Omega \cong \mathrm{Cte} . \mathrm{d} \Omega,
$$

where $I(\theta)$ is the intensity of the source in the $\theta$ direction, and $\mathrm{d} \Omega$ the elementary solid aperture angle.

We need to evaluate the flux measured with a curved sample compared to that obtained with a flat one, so the solid angular aperture has to be considered.

As a consequence of the change of the image source position, the aperture of the emergent beam varies, depending on the position and width of the stop apertures in the object and in the image space (Fig. 9). Because the position of the sagital focus $S_{1}$ does not vary, we can consider only the variation of the linear aperture angle $\alpha$ in the plane of incidence, in order to determine the relative variation of the measured flux when the sample curvature varies.

If $\alpha_{0}$ is the aperture of the incident beam, $\alpha_{1}$ is the aperture of the emergent beam. the intensity of which is measured by the detector. For a flat sample, the measured intensity is proportional to the ratio $\min \left(\alpha_{0}, \alpha_{1}\right) / \alpha_{0}$, while for a curved sample this ratio becomes $\alpha_{3} / \alpha_{2}$, where $\alpha_{2}$ carries the same amount of energy as $\alpha_{0}$.

So the product :

$$
\left(\alpha_{3} / \alpha_{2}\right) *\left(\alpha_{0} / \min \left(\alpha_{0}, \alpha_{1}\right)\right)
$$

gives the intensity measured with a curved sample, with respect tot that obtained from a flat sample on the same instrument.

Looking at figure 9 , it has to be noted, that in the case where the position of the source image is close to the sample, the emergent beam is very divergent, and only a small part of the incident energy is detected. So, refering to the abacus of figure 8 which gives the position of the image source, it can be explained why, in some cases, the reflected beam becomes weak and flat and can lead to errors in the procedure of adjustment. 

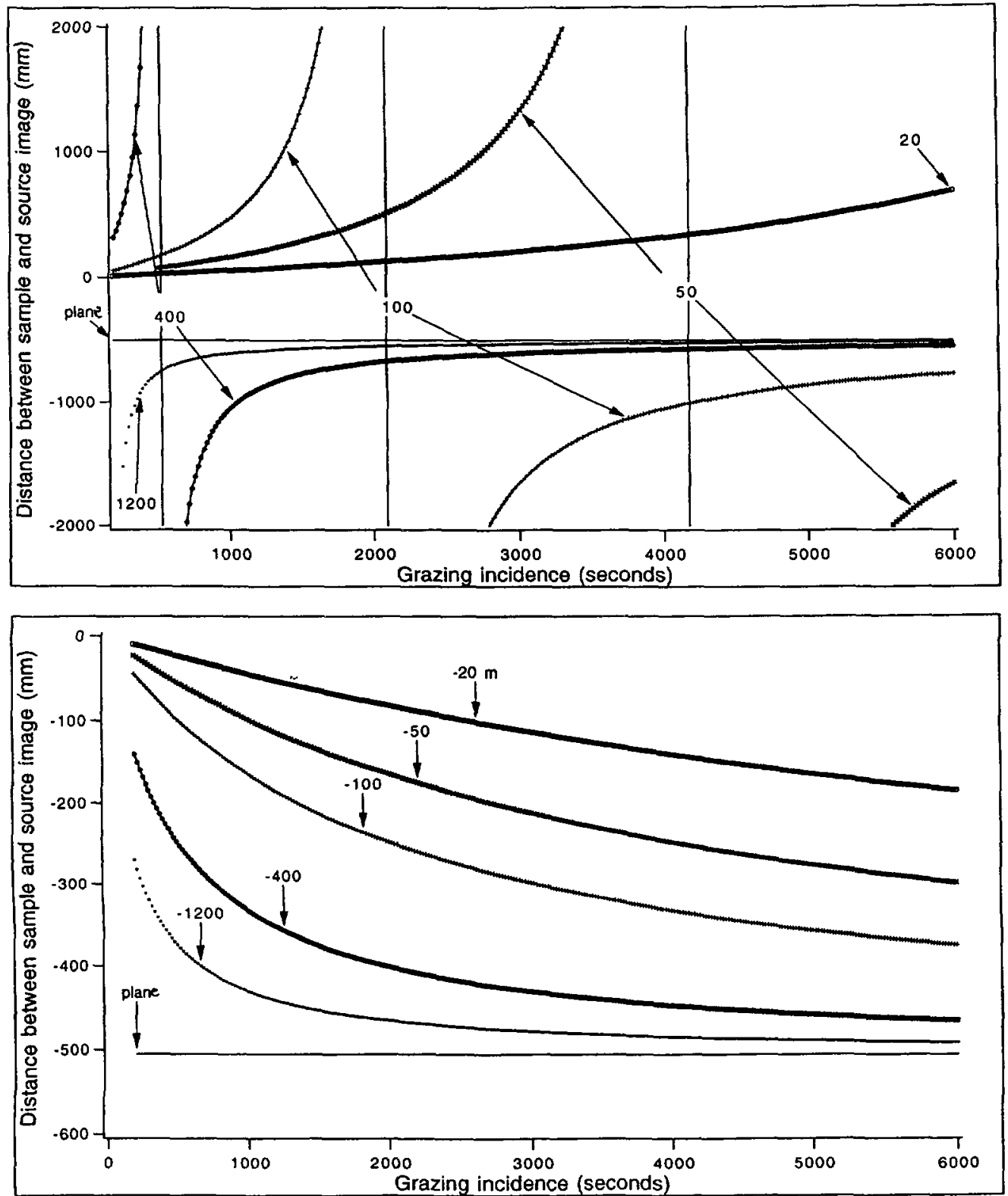

Fig. 8. - Variation of the distance between the source image and the sample is. grazing incidence for IOTA set-up.

The detailed calculation of the above geometrical factor involves the knowledge of the position and width of all the slits, as well as the geometrical parameters of the sample (curvature and size). A code of calculation of this coefficient was made. The theoretical results for IOTA set-up are compared with the experimental ones. In the plateau region, the effect is more visible with a linear scale for the intensity (Fig. 10). The misalignment effect during the adjustment procedure due to the curvature can explain the discrepancies that appear between the results, in particular the significant shift of the apparent critical angle.

It is shown that for $|R|<400 \mathrm{~m}$ the reflectivity measurement becomes defective. 

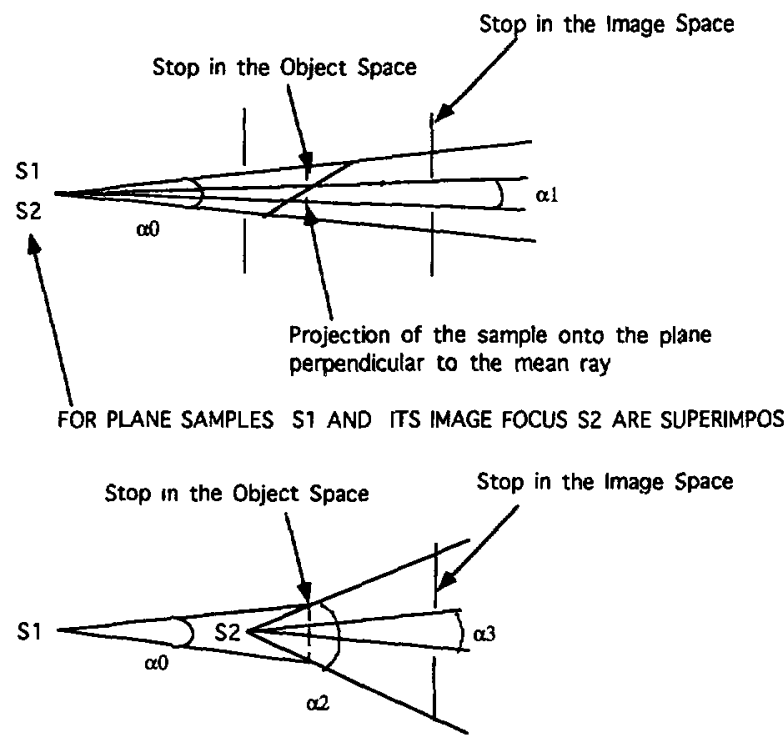

FOR CURVED SAMPLES THE INCIDENT BEAM THE APERTURE OF WTCH IS $\alpha 0$ SPREAD INTO $\alpha 2$ AND ONLY THE PART $\alpha 3$ FALLS ON THE DETECTOR

Fig. 9. - Variation of the aperture of the emergent beam with the position of the source image.
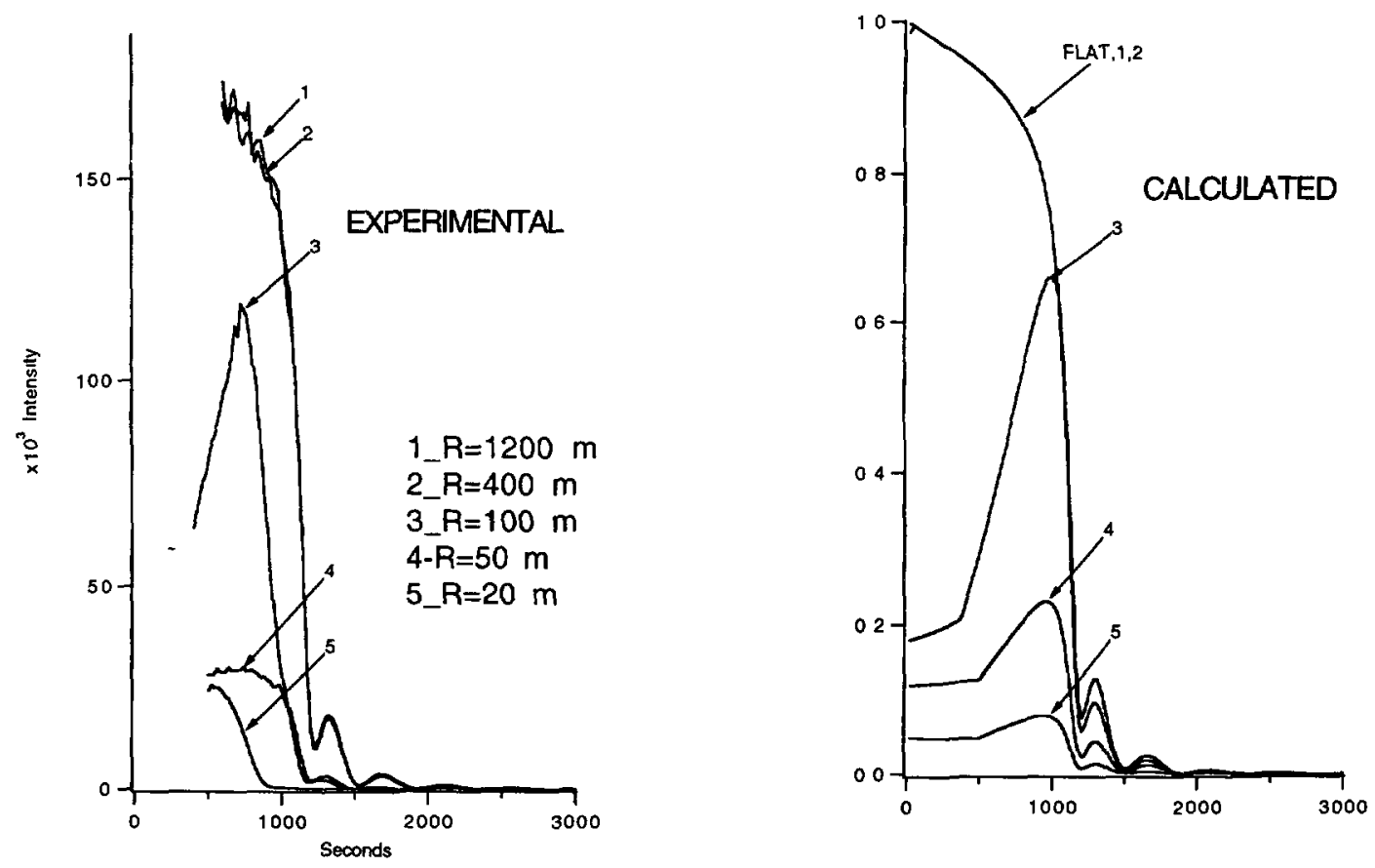

Fig. 10. - Comparıson between experimental and calculated reflectivity curves obtained with concave and convex samples on IOTA set-up. For comparison, the intensities are normalized for the $1200 \mathrm{~m}$ curved sample. Due to a mechanical constraint, the experimental data cannot reach the zero grazing angle. 


\section{Conclusion.}

For a given apparatus, it is possible to use this model in order to predict the reflected intensity in the plateau region, if the curvature and size of the sample are known, in particular in the plane of incidence.

One of the conditions for the validity of this calculation is that the adjustment of the apparatus can be achieved despite the curvature effect.

Another condition is that the real shape of the sample is not far from a sphere.

\section{Acknowledgements.}

This work was supported by the European BCR Commission within the NAMIX project. The author wish to thank F. Sacchetti and G. Gagliardi (SIV, Italy) for the coating of the curved samples, and B. Pardo and J. P. Chauvineau for the critical reading of this paper.

\section{References}

[1] Nevot L., Pardo B., Corno C., Analyse des matériaux stratifiés par réflectométrie X, Analysis 16 (1988) 381-388.

[2] Bridou F., Pardo B., Automatic characterization of layers stacks from reflectivity measurements. Application to the study of the validity conditions of the grazing X-rays reflectometry, $J$ Opt. (Paris) 21 (1990) 183-191.

[3] Nevot L., Croce P., Caractérisation des surfaces par réflexion rasante de rayons X. Application à l'étude du polissage de quelques verres silicates. Rev. Phys. Appl 15 (1980) 761-779.

[4] Maréchal A., Imagerie géométrıque, Aberrations (Edition de la Revue d'Optique, 1952). 